\title{
Finite-Time Synchronization for Uncertain Master-Slave Chaotic System via Adaptive Super Twisting Algorithm
}

\author{
P. Siricharuanun ${ }^{1}$ and C. Pukdeboon ${ }^{2}$ \\ ${ }^{1}$ Department of Mathematics, Faculty of Science, Kasetsart University, Bangkok 10900, Thailand \\ ${ }^{2}$ Department of Mathematics, Faculty of Applied Science, King Mongkut's University North Bangkok, Bangkok 10800, Thailand
}

Correspondence should be addressed to P. Siricharuanun; fscispns@ku.ac.th

Received 23 March 2016; Accepted 2 June 2016

Academic Editor: Marius-F. Danca

Copyright (c) 2016 P. Siricharuanun and C. Pukdeboon. This is an open access article distributed under the Creative Commons Attribution License, which permits unrestricted use, distribution, and reproduction in any medium, provided the original work is properly cited.

\begin{abstract}
A second-order sliding mode control for chaotic synchronization with bounded disturbance is studied. A robust finite-time controller is designed based on super twisting algorithm which is a popular second-order sliding mode control technique. The proposed controller is designed by combining an adaptive law with super twisting algorithm. New results based on adaptive super twisting control for the synchronization of identical Qi three-dimensional four-wing chaotic system are presented. The finite-time convergence of synchronization is ensured by using Lyapunov stability theory. The simulations results show the usefulness of the developed control method.
\end{abstract}

\section{Introduction}

Synchronization of chaotic system has been of increasing interest in recent years owing to its effective applications in secure communication, power convertors, biological systems, information processing, and chemical reactions $[1,2]$. A fundamental concept for chaos synchronization is to use the outputs of the master system to control the outputs of the slave system so that the states of the slave system track the states of master system. In practice, it is difficult to know the parameters of a chaotic system precisely and external disturbance always occurs in the system. Thus, synchronization of chaotic system in the presence of parameter uncertainties and external disturbances is effectively crucial in applications. Various nonlinear control methods have been proposed to deal with the problem of synchronization of uncertain chaotic systems such as adaptive control [3], passive control [4], sliding mode control $[5,6]$, backstepping control $[7,8]$, and fuzzy control [9].

Sliding mode control (SMC) $[10,11]$ is an effective nonlinear control method to deal with a system with uncertainties and external disturbance. However, there are two main drawbacks of sliding mode control. First, the convergence of system states to the equilibrium point is asymptotical, so the system states cannot converge to the equilibrium point within a finite time. The second drawback is the chattering phenomenon. Second-order sliding mode control (SOSMC) [12-14] is the enhanced SMC method which is developed to maintain good properties of SMC and reduce the chattering effect. Moreover, the recent SOSMC is designed based on the finite-time stability $[15,16]$. This can improve the convergence speed of SMC and keep the desired properties of SMC.

The aim of this paper is to design a robust finite-time feedback control for chaotic synchronization. As is known, the super twisting algorithm is in a class of second-order SMC and is widely used in many practical applications [1719]. Moreover, to deal with uncertainties and disturbance, the adaptive tuning law is combined with the super twisting algorithm. This adaptive law is used to update the controller gains and this relaxes the requirement of information of the bound of uncertainties and disturbances. The resulting controller is called adaptive-gain super twisting controller (AGSTC).

The rest of this paper is organized as follows. In Section 2.1, the synchronization problem is formulated and concepts and lemmas of finite-time stability are given. Section 2.2 presents the controller design for the synchronization problem via SMC. In Section 2.3, a robust finite-time 
controller design is proposed. The proposed adaptive super twisting controller is developed to achieve finite-time synchronization. Section 2.4 discusses the synchronization of identical Qi four-wing chaotic system. Section 3 presents the simulation results. Conclusions are provided in Section 4.

\section{Materials and Methods}

2.1. System Description and Problem Statement. Consider the chaotic system described by the following:

master system:

$$
\dot{x}=A x+f(x)+d_{x},
$$

slave system:

$$
\dot{y}=A y+f(y)+u+d_{y},
$$

where $x \in R^{n}$ and $y \in R^{n}$ are the states of the master and slave systems, $A$ is the $n \times n$ matrix of the system parameters, $f: R^{n} \rightarrow R^{n}$ is the nonlinear part of the system, $u \in R^{m}$ is the controller to be designed, and $d_{x}, d_{y} \in R^{m}$ are external disturbances for master and slave systems, respectively. We define the synchronization error as

$$
e=y-x
$$

From master system (1) and slave system (2), we obtain the error dynamic as

$$
\dot{e}=A e+\eta(x, y)+u+\widetilde{d}
$$

where $\eta(x, y)=f(y)-f(x)$ and $\tilde{d}=d_{y}-d_{x}$.

We consider the master and slave chaotic systems described by (1) and (2), respectively. The aim is to find a controller $u$ so that the error state $e$ converges to zero in a finite time represented by a constant $T=T(e(0))>0$. In other words, we need $\lim _{t \rightarrow T}\|e(t)\|=0$ and $\|e(t)\| \equiv 0$, when $t \geq T$. This implies that the chaos synchronization between chaotic systems (1) and (2) is realized in the finite time $T$. We now restate the concepts related to finite-time stability presented by Bhat and Bernstein $[15,16]$.

Lemma 1 (Bhat and Bernstein [15]). Consider the system

$$
\dot{x}=f(x), \quad f(0)=0, x \in R^{n},
$$

where $f: D \rightarrow R^{n}$ is continuous on an open neighborhood $D \subset R^{n}$. Assume that there is a continuous differential positive definite function $V(x): D \rightarrow R$ and real numbers $p>0$ and $0<\eta<1$, such that

$$
\dot{V}(x)+p V^{\eta}(x) \leq 0, \quad \forall x \in D .
$$

Then, the origin of system (5) is a locally finite-time stable equilibrium, and the settling time, depending on the initial state $x(0)=x_{0}$, satisfies

$$
T\left(x_{0}\right) \leq \frac{V^{1-\eta}\left(x_{0}\right)}{p(1-\eta)} .
$$

In addition, if $D=R^{n}$ and $V(x)$ is also radially unbounded, then the origin is a globally finite-time stable equilibrium of systems (5).
Lemma 2 (Yu et al. [20]). For any numbers $\lambda_{1}>0, \lambda_{2}>0$, and $0<\omega<1$, an extended Lyapunov condition of finite-time stability can be given in the form of fast terminal sliding mode as

$$
\dot{V}(x)+\lambda_{1} V(x)+\lambda_{2} V^{\varrho}(x) \leq 0,
$$

where the settling time can be estimated by

$$
T_{r} \leq \frac{1}{\lambda_{1}(1-\omega)} \ln \left(\frac{\lambda_{1} V^{1-\Phi}\left(x_{0}\right)+\lambda_{2}}{\lambda_{2}}\right) .
$$

2.2. Synchronization via Sliding Mode Controller. We define the sliding variable defined as

$$
s=C e,
$$

where $C=\left[\begin{array}{llll}c_{1} & c_{2} & \cdots & c_{n}\end{array}\right]$ is a $1 \times n$ constant matrix and $e=$ $\left[\begin{array}{llll}e_{1} & e_{2} & \cdots & e_{n}\end{array}\right]^{T}$ is the synchronization error. In the SMC, the motion of system (4) is driven to the sliding surface defined by

$$
s(e)=\left\{x \in R^{n} \mid s(e)=0\right\},
$$

which is required to be invariant under the flow of the error dynamic (4). The necessary condition for state trajectory $e(t)$ to stay on the sliding manifold $s$ is $s \dot{s}<0$. We ignore the disturbance vector $\tilde{d}$ and apply the constant plus proportional rate reaching law:

$$
\dot{s}=-q \operatorname{sign}(s)-k s,
$$

where $\operatorname{sign}(s)$ is the sign function and the constant gains $q>0$ and $k>0$ are determined such that the sliding condition is satisfied. The proposed SMC is designed as

$$
u=-C^{-1} q \operatorname{sign}(s)-k e-A e-\eta(x, y) .
$$

In the following theorem, under controller (13) we can ensure that the synchronization occurs asymptotically.

Theorem 3. Master system (1) and slave system (2) are globally and asymptotically synchronized for all initial conditions $x(0), y(0) \in R^{n}$ by the feedback control law (13).

Proof. Substituting (13) into (4), we obtain

$$
\dot{e}=-C^{-1} q \operatorname{sign}(s)-k e+\tilde{d} .
$$

We consider the Lyapunov function

$$
V_{1}=\frac{1}{2} s^{2}
$$

which is positive definite function on $R$. Differentiating (15), we obtain

$$
\dot{V}_{1}=s \dot{s} .
$$

Substituting (12) into (16), we obtain

$$
\dot{V}_{1}=s(-q \operatorname{sign}(s)-k s) .
$$


Using the fact that $\operatorname{sign}(s)=|s| / s$, one has

$$
\dot{V}_{1}=-q|s|-k s^{2}<0, \quad \forall e \neq 0 .
$$

Obviously, $\dot{V}_{1}$ is negative definite. Thus, the error state $e$ globally and asymptotically reaches the sliding surface $s=$ 0 .

2.3. Adaptive-Gain Super Twisting Controller. The super twisting control law is the most powerful second-order continuous sliding mode control algorithms. It generates the continuous control function that drives the sliding variable and its derivative to zero in finite time. Next, we add an adaptive law to the classical super twisting algorithm to tune the controller gains and avoid knowledge of upper bound of the vector $\tilde{d}$.

We use the sliding variable $e$ defined by (10) and introduce a new reaching law as

$$
\dot{s}=-k_{1}|s|^{1 / 2} \operatorname{sign}(s)-k_{2} \int_{0}^{t} \operatorname{sign}(s(\tau)) d \tau,
$$

where $k_{1}$ and $k_{2}$ are positive gains defined as $k_{1}=\lambda_{1} \mu L(t)$ and $k_{2}=\lambda_{2}\left(\mu^{2} L^{2}(t) / 2\right)$, where $\lambda_{1}$ and $\lambda_{2}$ are positive constants and $L(t)$ is updated by

$$
\dot{L}(t)= \begin{cases}l(t), & s_{i} \neq 0 \\ 0, & s_{i}=0\end{cases}
$$

with a continuous function $l(t)>0$.

Considering the error dynamic (4), the adaptive super twisting controller is designed as

$$
\begin{aligned}
u= & -\eta(x, y)-(C)^{-1} \\
& \cdot\left[C A e+k_{1}|s|^{1 / 2} \operatorname{sign}(s)-k_{2} \int_{0}^{t} \operatorname{sign}(s(\tau)) d \tau\right] .
\end{aligned}
$$

Substituting (21) into (4), one obtains

$$
\dot{s}=-k_{1}|s|^{1 / 2} \operatorname{sign}(s)-k_{2} \int_{0}^{t} \operatorname{sign}(s(\tau)) d \tau+\xi,
$$

where $\xi=C \tilde{d}$.

Let us define

$$
\begin{aligned}
z_{1} & =s, \\
z_{2} & =-\lambda_{2} \frac{\mu^{2} L^{2}(t)}{2} \int_{0}^{t} \operatorname{sign}(s(\tau)) d \tau+\xi \\
\dot{\xi}(t) & =\delta(t) .
\end{aligned}
$$

Then, (22) can be written as

$$
\begin{aligned}
& \dot{z}_{1}=-\lambda_{1} \mu L(t)\left|z_{1}\right|^{1 / 2} \operatorname{sign}\left(z_{1}\right)+z_{2}, \\
& \dot{z}_{2}=-\lambda_{2} \frac{\mu^{2} L^{2}(t)}{2} \operatorname{sign}\left(z_{1}\right)+\delta(t) .
\end{aligned}
$$

Next, for system (24) under the following assumption, the proof of finite-time convergence to the origin is given.
Assumption 4. The new disturbance $\xi(t)$ and its first-time derivative $\delta(t)$ are bounded; that is, $|\xi(t)| \leq D_{1}$ and $|\delta(t)| \leq$ $D_{2}$, where $D_{1}$ and $D_{2}$ are positive constants.

Theorem 5. Let Assumption 4 hold. With $\mu>0$ and $\lambda_{1}>0$ and $\lambda_{2}>0$ and $L(t)$ defined in (20), all states $\left(z_{1}\right.$ and $\left.z_{2}\right)$ of system (24) converge to the origin in finite time.

Proof. We first introduce the new vector $v=$ $\left[\left|z_{1}\right|^{1 / 2} \operatorname{sign}\left(z_{1}\right) \quad z_{2}\right]^{T}$. Its time derivative is given by

$$
\begin{aligned}
\dot{v} & =\frac{1}{2\left|z_{1}\right|^{1 / 2}}\left[\begin{array}{c}
-\lambda_{1} \mu L\left|z_{1}\right|^{1 / 2} \operatorname{sign}\left(z_{1}\right)+z_{2} \\
-\lambda_{2} \mu^{2} L^{2}\left|z_{1}\right|^{1 / 2} \operatorname{sign}\left(z_{1}\right)+2\left|z_{1}\right|^{1 / 2} \delta
\end{array}\right] \\
& =\frac{1}{2\left|z_{1}\right|^{1 / 2}}\left(\left[\begin{array}{cc}
-\lambda_{1} \mu & 1 \\
-\lambda_{2} \mu^{2} L^{2} & 0
\end{array}\right] v+\left[\begin{array}{c}
0 \\
2\left|z_{1}\right|^{1 / 2} \delta
\end{array}\right]\right) .
\end{aligned}
$$

Next, we change variable as $\varsigma=\Gamma^{-1} v$ and obtain

$$
\varsigma=\left[\begin{array}{ll}
\frac{v_{1}}{L} & \frac{v_{2}}{L^{2}}
\end{array}\right]^{T}
$$

where $\Gamma=\left[\begin{array}{cc}L & 0 \\ 0 & L^{2}\end{array}\right]$. The derivative of $\varsigma$ is

$$
\begin{aligned}
& \dot{\zeta}=\Gamma^{-1} \dot{v}+\dot{\Gamma}^{-1} v \\
& =\Gamma^{-1}\left(\frac{1}{2\left|z_{1}\right|^{1 / 2}}\left[\begin{array}{cc}
-\lambda_{1} \mu L & 1 \\
-\lambda_{2} \mu^{2} L^{2} & 0
\end{array}\right] v+\left[\begin{array}{c}
0 \\
2\left|z_{1}\right|^{1 / 2} \delta
\end{array}\right]\right) \\
& +\left[\begin{array}{r}
-\frac{v_{1}}{L^{2}} \\
-\frac{v_{2}}{L^{3}}
\end{array}\right] \dot{L} \\
& =\frac{1}{2\left|z_{1}\right|^{1 / 2}}\left(\left[\begin{array}{cc}
-\lambda_{1} \mu L & L \\
-\lambda_{2} \mu^{2} L & 0
\end{array}\right] \varsigma+\Gamma^{-1}\left[\begin{array}{c}
0 \\
2\left|z_{1}\right|^{1 / 2} \delta
\end{array}\right]\right) \\
& -\frac{\dot{L}}{L}\left[\begin{array}{ll}
1 & 0 \\
0 & 2
\end{array}\right]\left[\begin{array}{c}
\frac{v_{1}}{L} \\
\frac{v_{2}}{L^{2}}
\end{array}\right] \\
& =\frac{1}{2\left|z_{1}\right|^{1 / 2}}\left(L\left[\begin{array}{cc}
-\lambda_{1} \mu & 1 \\
-\lambda_{2} \mu^{2} & 0
\end{array}\right] \varsigma+\Gamma^{-1}\left[\begin{array}{c}
0 \\
2\left|z_{1}\right|^{1 / 2} \delta
\end{array}\right]\right) \\
& -\frac{\dot{L}}{L}\left[\begin{array}{ll}
1 & 0 \\
0 & 2
\end{array}\right]\left[\begin{array}{c}
\frac{v_{1}}{L} \\
\frac{v_{2}}{L^{2}}
\end{array}\right] \\
& =\frac{1}{2\left|z_{1}\right|^{1 / 2}}\left(L A \varsigma+\Gamma^{-1} \varphi\right)-\frac{\dot{L}}{L} N \varsigma,
\end{aligned}
$$


where

$$
\begin{aligned}
& A=\left[\begin{array}{cc}
-\lambda_{1} \mu & 1 \\
-\lambda_{2} & 0
\end{array}\right], \\
& \varphi=\left[\begin{array}{c}
0 \\
2\left|z_{1}\right|^{1 / 2} \delta
\end{array}\right], \\
& N=\left[\begin{array}{ll}
1 & 0 \\
0 & 2
\end{array}\right] .
\end{aligned}
$$

We now construct the Lyapunov function by extending the ideas of Moreno and Osorio [21].

Let the Lyapunov function be chosen as

$$
V_{2}=\varsigma^{T} P \varsigma
$$

where $P$ is the solution of the Lyapunov equation:

$$
A^{T} P+P A=-Q
$$

If the gains $\lambda_{1}$ and $\lambda_{2}$ are chosen such that the matrix $A$ is Hurwitz and arbitrary symmetric positive definite matrix $Q$ is selected, then the solution $P$ is unique and symmetric positive definite. Finding the derivative of $V_{2}$, we obtain

$$
\dot{V}_{2}=\frac{1}{2\left|z_{1}\right|^{1 / 2}}\left(2 L \varsigma^{T} P A \varsigma+2 \varsigma^{T} P \Gamma^{-1} \varphi\right)-\frac{2 \dot{L}}{L} \varsigma^{T} P N \varsigma .
$$

We consider the term $\Gamma^{-1} \varphi$, and one obtains

$$
\begin{aligned}
\Gamma^{-1} \varphi & =\frac{2}{L^{2}} \delta\left|z_{1}\right|^{1 / 2} \\
& \leq \frac{2}{L}|\delta| \sqrt{\left(\frac{\left|z_{1}\right|^{1 / 2} \operatorname{sign}(z)}{L}\right)^{2}+\frac{z_{2}^{2}}{L^{4}}} \leq \frac{2}{L} D_{2}\|\varsigma\| .
\end{aligned}
$$

Thus, $\dot{V}_{2}$ in (31) becomes

$$
\begin{aligned}
\dot{V}_{2} & \\
\leq & -\frac{1}{2}\left|z_{1}\right|^{-1 / 2}\left[L \varsigma^{T}(Q) \varsigma-\frac{4}{L} D_{2} \lambda_{\max }(P)\|\varsigma\|^{2}\right] \\
& -\frac{2 \dot{L}}{L} \varsigma^{T}(P N) \varsigma \\
\leq & -\frac{1}{2}\left|z_{1}\right|^{-1 / 2}\left[L \lambda_{\min }(Q)\|\varsigma\|^{2}-\frac{4}{L} D_{2} \lambda_{\max }(P)\|\varsigma\|^{2}\right] \\
& -\frac{2 \dot{L}}{L} \lambda_{\min }(R)\|\varsigma\|^{2} \\
\leq & -\frac{1}{2}\left|z_{1}\right|^{-1 / 2}\left[L \lambda_{\min }(Q)-\frac{4}{L} D_{2} \lambda_{\max }(P)\right]\|\varsigma\|^{2} \\
& -\frac{2 \dot{L}}{L} \lambda_{\min }(R)\|\varsigma\|^{2} .
\end{aligned}
$$

Using $-1 /\left|z_{1}\right|^{1 / 2} \leq-1 /\|\varsigma\|$, we obtain

$$
\begin{aligned}
\dot{V}_{2} \leq & -\frac{1}{2\|\varsigma\|}\left[L \lambda_{\min }(Q)-\frac{4}{L} D_{2} \lambda_{\max }(P)\right]\|\varsigma\|^{2} \\
& -\frac{2 \dot{L}}{L} \lambda_{\min }(R)\|\varsigma\|^{2} \\
\leq & -\frac{1}{2}\left[L \lambda_{\min }(Q)-\frac{4}{L} D_{2} \lambda_{\max }(P)\right]\|\varsigma\| \\
& -\frac{2 \dot{L}}{L} \lambda_{\min }(R)\|\varsigma\|^{2} \\
\leq & -\frac{1}{2}\left[L \lambda_{\min }(Q)-\frac{4}{L} D_{2} \lambda_{\max }(P)\right] \frac{V_{2}^{1 / 2}}{\sqrt{\lambda_{\max }(P)}} \\
& -\frac{2 \dot{L}}{L}\left[\frac{\lambda_{\min }(R)}{\lambda_{\max }(P)}\right] V_{2},
\end{aligned}
$$

where $R=P N$. It is observed that there exists a time $t_{0}$, where the gain $L(t)$ is sufficiently large such that $\dot{V}_{2}<0$ is attained. Therefore, by Lemma 2 , the states $\varsigma_{1}$ and $\varsigma_{2}$ converge to zero in finite time. This implies the finite-time convergence to zero in states $z_{1}$ and $z_{2}$. As a consequence the gain $L(t)$ will stop growing in finite time and it will remain bounded.

2.4. Synchronization of Identical Qi Four-Wing Chaotic System. Qi four-wing chaotic systems are described as follows:

master system:

$$
\begin{aligned}
& \dot{x}_{1}=(a+\Delta a)\left(x_{2}-x_{1}\right)+\varepsilon x_{2} x_{3}+d x_{1} \\
& \dot{x}_{2}=c x_{1}+d x_{2}-x_{1} x_{3}+d x_{2} \\
& \dot{x}_{3}=-(b+\Delta b) x_{3}+x_{1} x_{2}+d x_{3},
\end{aligned}
$$

slave system:

$$
\begin{aligned}
& \dot{y}_{1}=(a+\Delta a)\left(y_{2}-y_{1}\right)+\varepsilon y_{2} y_{3}+u_{1}+d y_{1} \\
& \dot{y}_{2}=c y_{1}+d y_{2}-y_{1} y_{3}+u_{2}+d y_{2} \\
& \dot{y}_{3}=-(b+\Delta b) y_{3}+y_{1} y_{2}+u_{3}+d y_{3},
\end{aligned}
$$

where $x_{i}$ and $y_{i}(i=1,2,3)$ are state variables of the master and slave systems, respectively.

Note that systems (35) and (36) are obtained by considering (1) and (2), where $A, x, y, f(x), f(y), d_{x}$, and $d_{y}$ are defined as follows:

$$
\begin{aligned}
& A=\left[\begin{array}{ccc}
-a & a & 0 \\
c & d & 0 \\
0 & 0 & -b
\end{array}\right], \\
& x=\left[\begin{array}{l}
x_{1} \\
x_{2} \\
x_{3}
\end{array}\right],
\end{aligned}
$$



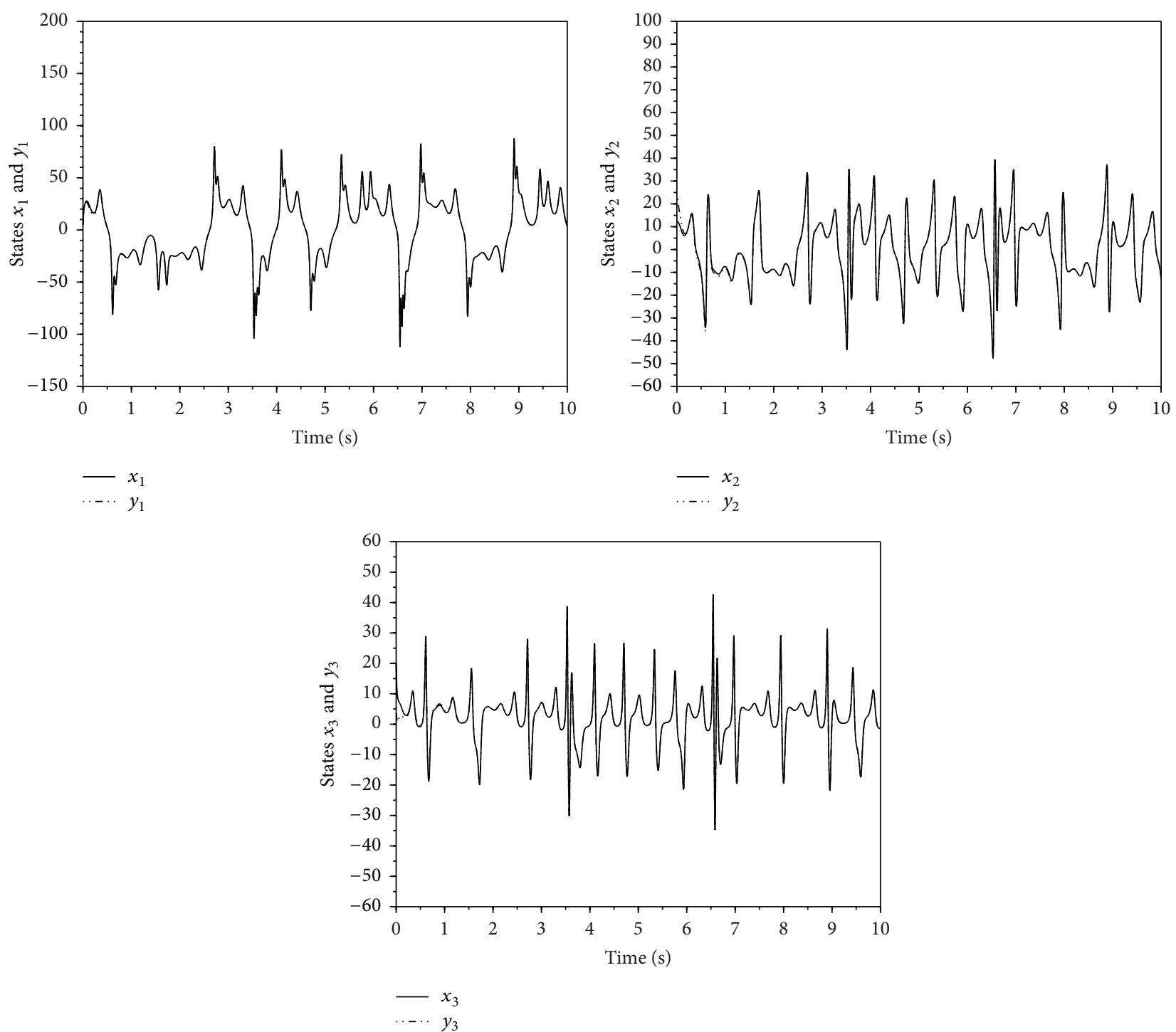

FIGURE 1: Synchronization of identical Qi four-wing system for SMC.

$$
\begin{aligned}
& y=\left[\begin{array}{l}
y_{1} \\
y_{2} \\
y_{3}
\end{array}\right] \\
& f(x)=\left[\begin{array}{c}
\varepsilon x_{2} x_{3} \\
-x_{1} x_{3} \\
x_{1} x_{2}
\end{array}\right], \\
& f(y)=\left[\begin{array}{c}
\varepsilon y_{2} y_{3} \\
-y_{1} y_{3} \\
y_{1} y_{2}
\end{array}\right], \\
& \Delta A=\left[\begin{array}{ccc}
-\Delta a & \Delta a & 0 \\
0 & 0 & 0 \\
0 & 0 & -\Delta b
\end{array}\right],
\end{aligned}
$$$$
\begin{gathered}
d_{x}=\left[\begin{array}{l}
d x_{1} \\
d x_{2} \\
d x_{3}
\end{array}\right], \\
d_{y}=\left[\begin{array}{l}
d y_{1} \\
d y_{2} \\
d y_{3}
\end{array}\right], \\
u=\left[\begin{array}{l}
u_{1} \\
u_{2} \\
u_{3}
\end{array}\right] .
\end{gathered}
$$$$
\text { In (36), the control laws } u_{1}, u_{2}, u_{3} \text { can be designed together }
$$
in the form of vector $u$ which is more convenient for our 

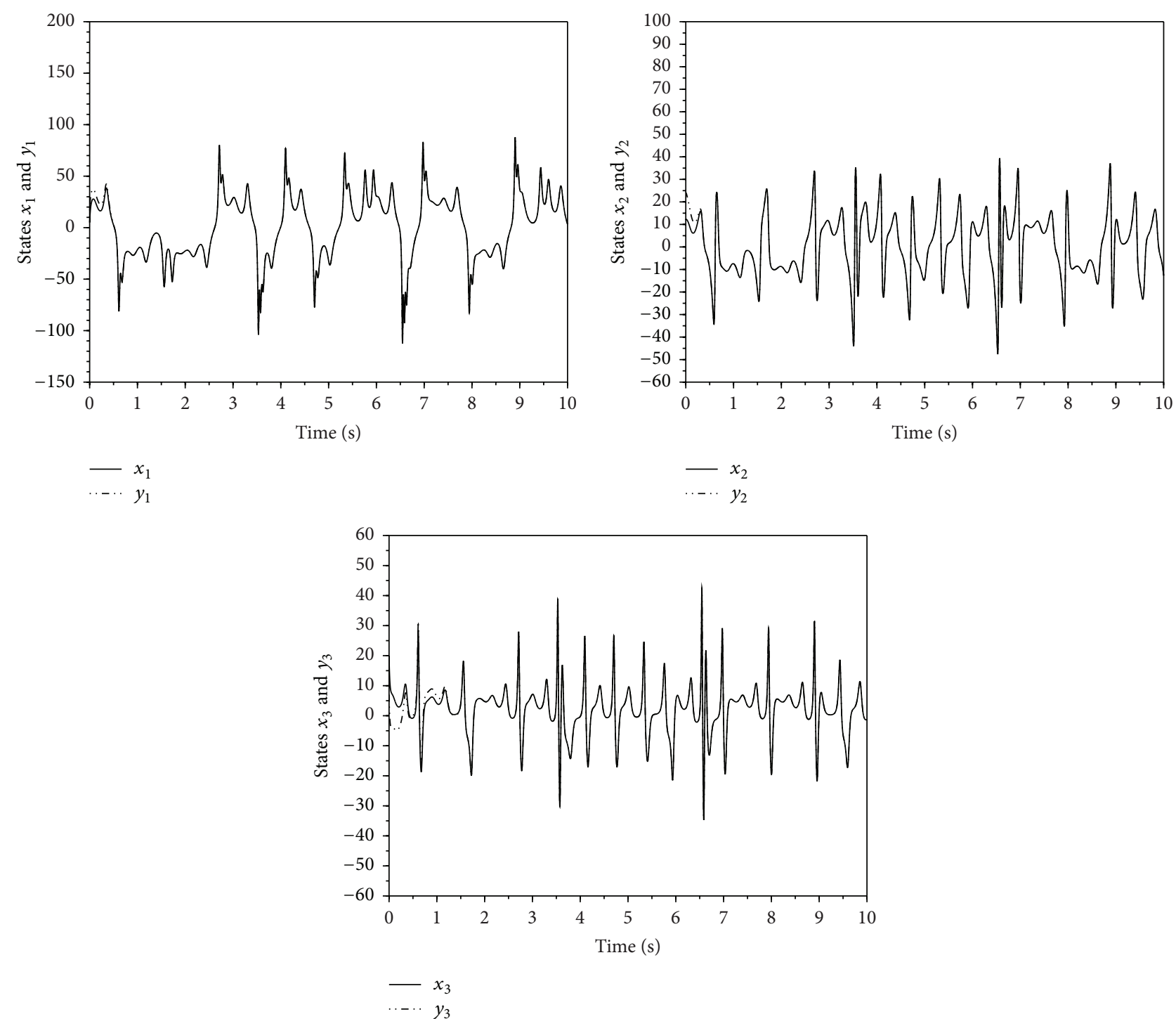

FIGURE 2: Synchronization of identical Qi four-wing system for AGSTC.

proposed method. The parameters $a, b, c, d, \varepsilon$ are positive constants. The synchronization error is defined by

$$
e_{i}=y_{i}-x_{i}, \quad(i=1,2,3)
$$

We obtained the error dynamics as

$$
\begin{aligned}
& \dot{e}_{1}=(a+\Delta a)\left(e_{2}-e_{1}\right)+\varepsilon\left(y_{2} y_{3}-x_{2} x_{3}\right)+u_{1} \\
& \dot{e}_{2}=c e_{1}+d e_{2}-y_{1} y_{3}+x_{1} x_{3}+u_{2} \\
& \dot{e}_{3}=-(b+\Delta b) e_{3}+y_{1} y_{2}-x_{1} x_{2}+u_{3} .
\end{aligned}
$$

We rewrite the error dynamics (39) as

$$
\dot{e}=A e+\eta(x, y)+u+\widetilde{d}
$$

where

$$
A=\left[\begin{array}{ccc}
-a & a & 0 \\
c & d & 0 \\
0 & 0 & -b
\end{array}\right]
$$

$$
e=\left[\begin{array}{l}
e_{1} \\
e_{2} \\
e_{3}
\end{array}\right]
$$

$$
\eta(x, y)=\left[\begin{array}{c}
\varepsilon\left(y_{2} y_{3}-x_{2} x_{3}\right) \\
-y_{1} y_{3}+x_{1} x_{3} \\
y_{1} y_{2}-x_{1} x_{2}
\end{array}\right],
$$




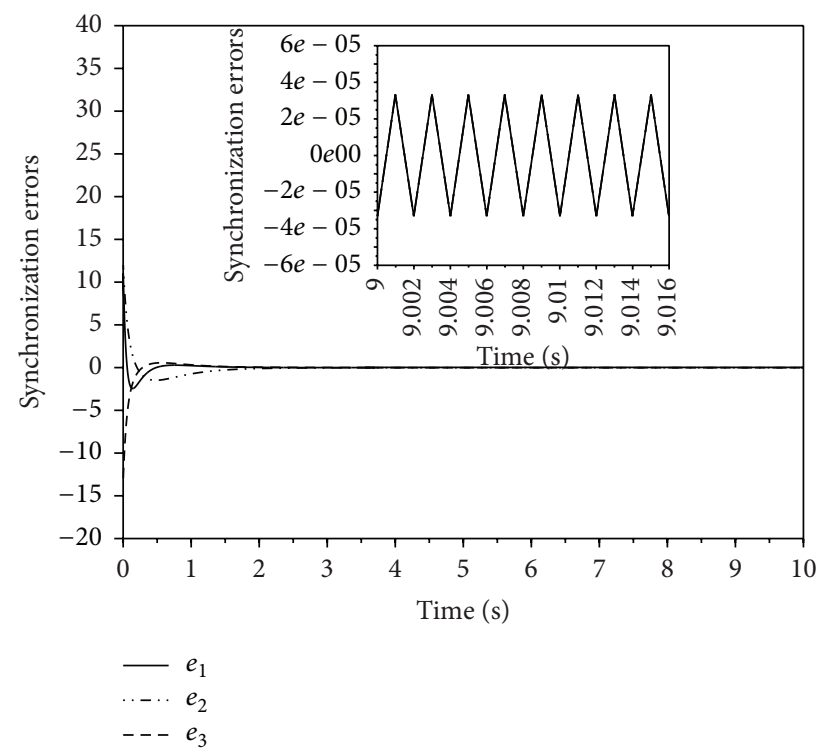

FIGURE 3: Synchronization errors of identical Qi four-wing system for SMC.

$$
\begin{aligned}
& u=\left[\begin{array}{l}
u_{1} \\
u_{2} \\
u_{3}
\end{array}\right], \\
& \tilde{d}=\Delta A e,
\end{aligned}
$$

with

$$
\Delta A=\left[\begin{array}{ccc}
-\Delta a & \Delta a & 0 \\
0 & 0 & 0 \\
0 & 0 & -\Delta b
\end{array}\right], \quad \Delta a=\Delta b=0.2 \sin t .
$$

\section{Results and Discussion}

In this section, numerical simulations are performed to compare the performances of sliding mode control (SMC) defined in (13) and adaptive-gain super twisting control (AGSTC) defined in (21).

The parameters for the chaotic systems (35) and (36) and the control laws (13) and (21) are set as $a=14, b=43, c=-1$, $d=16, \varepsilon=4, k=4, q=0.2, k_{1}=2.5 L(t), k_{2}=5\left(L^{2}(t) / 2\right)$, and

$$
\dot{L}(t)= \begin{cases}50, & \text { if }|s| \geq 0.0001 \\ 0 . & \text { otherwise }\end{cases}
$$

The initial values of the master system (1) are taken as $x_{1}(0)=$ $5, x_{2}(0)=12$, and $x_{3}(0)=20$ and initial values of the slave system (2) are taken as $y_{1}(0)=16, y_{2}(0)=24$, and $y_{3}(0)=7$.

The simulation results of synchronization under SMC and AGSTC are shown in Figures 1-8. As shown in Figures 1 and 2, for AGSTC the states of slave system quickly track the states of master system when compared with SMC. Similarly,

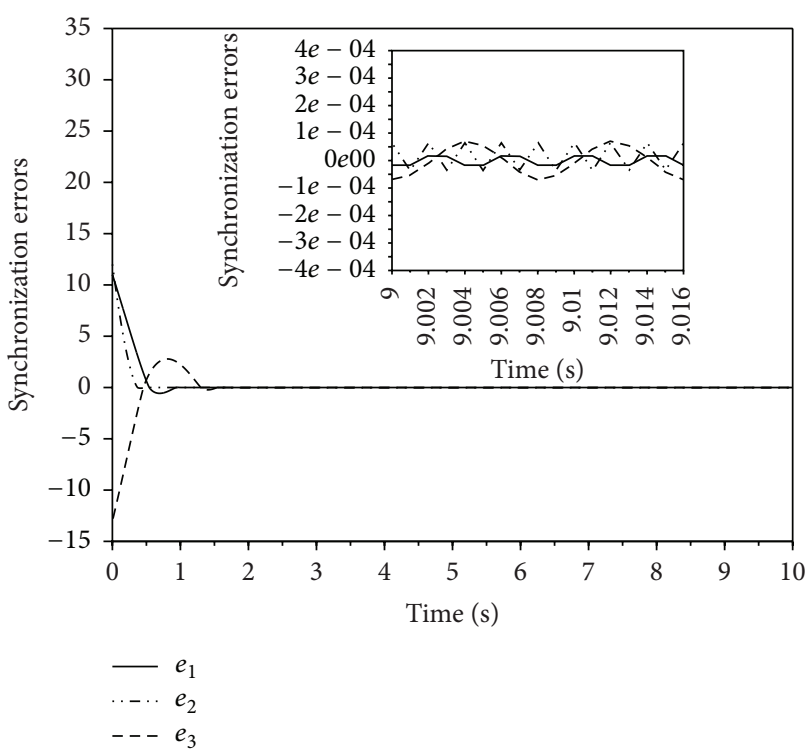

FIGURE 4: Synchronization errors of identical Qi four-wing system for AGSTC.

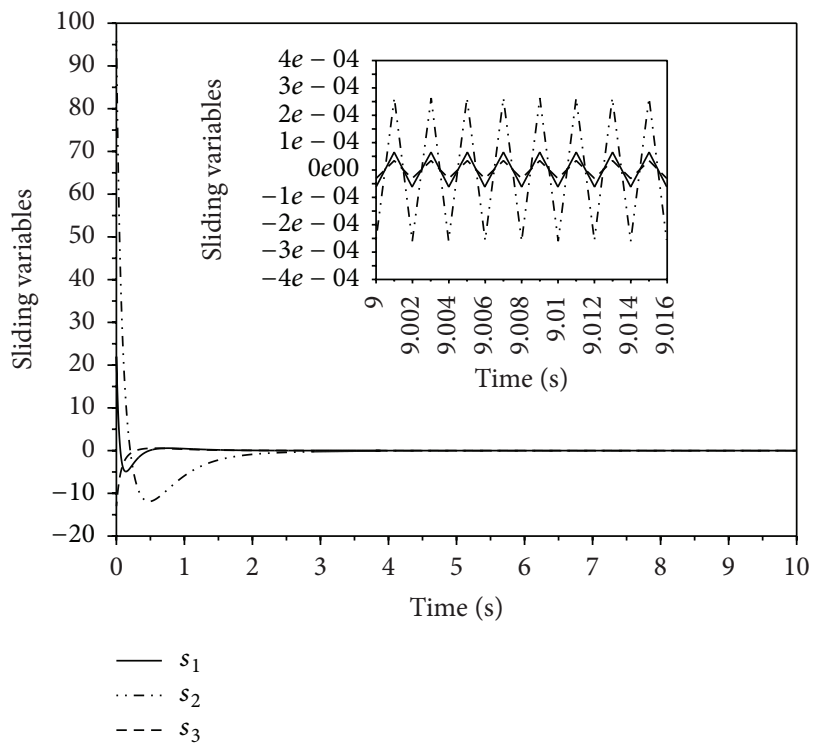

FIGURE 5: Sliding variables of identical Qi four-wing system for SMC.

Figures 3 and 4 show the error of synchronization in which AGSTC provides faster rate of convergence. For AGSTC, the synchronization error is larger than the one obtained by SMC because the control parameters $\lambda_{1}, \mu$, and $L(t)$ updated by (43) are chosen large enough to ensure the disturbance rejection ability. This leads to larger synchronization errors obtained by AGSTC as shown in Figures 3 and 4. From Figures 5 and 6, we can see that, for AGSTC, the sliding variables reach zero quicker. In Figures 7 and 8, the control inputs obtained by AGSTC are smoother than SMC. From these simulation results, it is clearly shown that AGSTC gives better results of synchronization. 


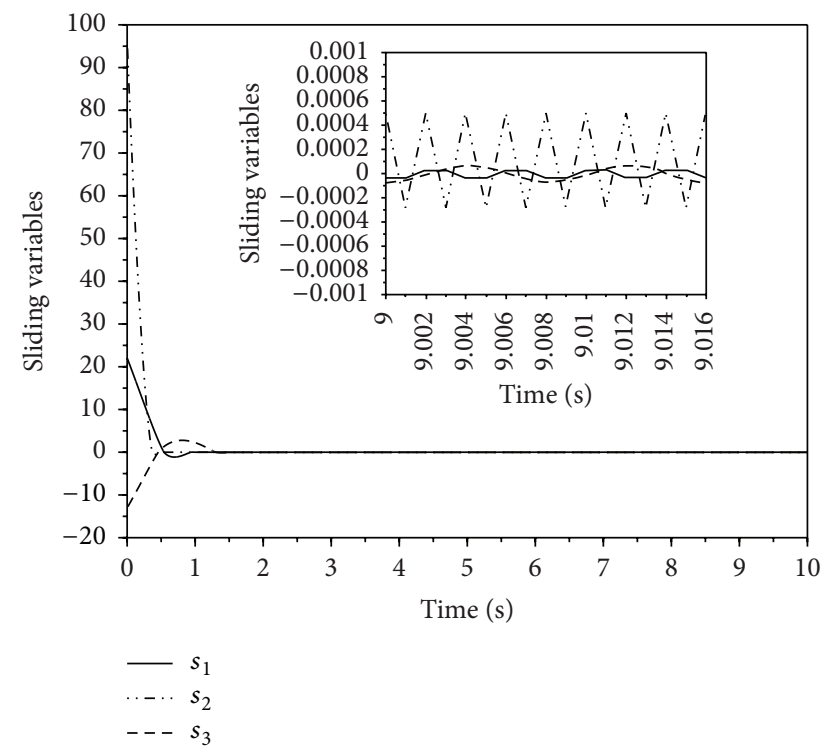

FIGURE 6: Sliding variables of identical Qi four-wing system for AGSTC.

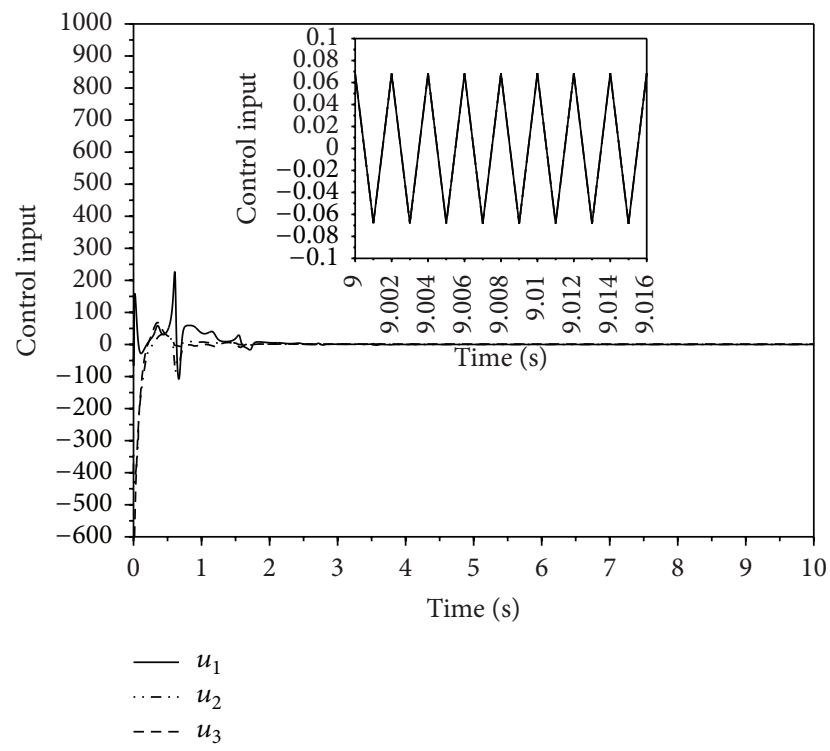

FIgURE 7: Control input of identical Qi four-wing system for SMC.

\section{Conclusions}

A robust finite-time controller has been successfully applied to synchronize identical Qi three-dimensional (3D) fourwing chaotic systems. The proposed control law is designed combining the super twisting algorithm with an adaptive tuning law. The finite-time convergence of synchronization error is proved using the Lyapunov stability theory. Numerical simulations are provided to validate synchronization results of the developed control method.

\section{Competing Interests}

The authors declare that they have no competing interests.

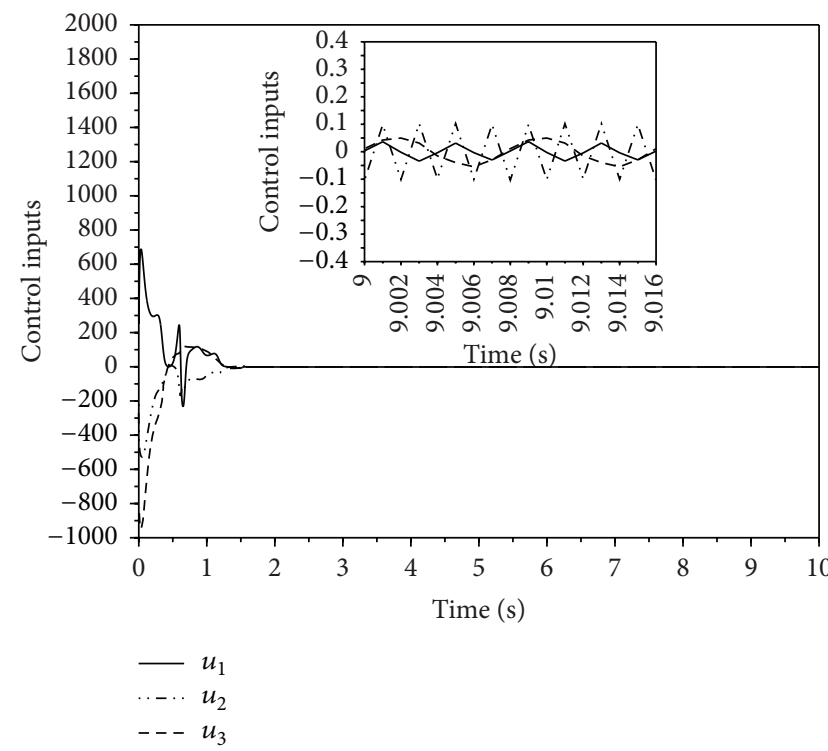

FIGURE 8: Control input of identical Qi four-wing system for AGSTC.

\section{References}

[1] T. Kapitaniak, Chaotic Oscillations in Mechanical Systems, Manchester University Press, New York, NY, USA, 1991.

[2] M. Lakshmanan and K. Murali, Chaos in Nonlinear Oscillators: Controlling and Synchronization, World Scientific, Singapore, 1996.

[3] S. Bowong, "Adaptive synchronization between two different chaotic dynamical systems," Communications in Nonlinear Science and Numerical Simulation, vol. 12, no. 6, pp. 976-985, 2007.

[4] F. Wang and C. Liu, "Synchronization of unified chaotic system based on passive control," Physica D, vol. 225, no. 1, pp. 55-60, 2007.

[5] H. Wang, Z. Han, Q. Xie, and W. Zhang, "Finite-time chaos synchronization of unified chaotic system with uncertain parameters," Communications in Nonlinear Science and Numerical Simulation, vol. 14, no. 5, pp. 2239-2247, 2009.

[6] H. Wang, Z.-Z. Han, Q.-Y. Xie, and W. Zhang, "Sliding mode control for chaotic systems based on LMI," Communications in Nonlinear Science and Numerical Simulation, vol. 14, no. 4, pp. 1410-1417, 2009.

[7] M. T. Yassen, "Controlling, synchronization and tracking chaotic Liu system using active backstepping design," Physics Letters, Section A: General, Atomic and Solid State Physics, vol. 360, no. 4-5, pp. 582-587, 2007.

[8] H.-H. Chen, G.-J. Sheu, Y.-L. Lin, and C.-S. Chen, "Chaos synchronization between two different chaotic systems via nonlinear feedback control," Nonlinear Analysis: Theory, Methods \& Applications, vol. 70, no. 12, pp. 4393-4401, 2009.

[9] H.-T. Yau and C.-S. Shieh, "Chaos synchronization using fuzzy logic controller," Nonlinear Analysis: Real World Applications, vol. 9, no. 4, pp. 1800-1810, 2008.

[10] V. I. Utkin, Sliding Modes in Control and Optimization, Communications and Control Engineering Series, Springer, Berlin, Germany, 1992. 
[11] C. Edwards, E. Fossas Colet, and L. Fridman, Eds., Advances in Variable Structure and Sliding Mode Control, Springer, Berlin, Germany, 2006.

[12] A. Levant, "Higher-order sliding modes, differentiation and output-feedback control," International Journal of Control, vol. 76, no. 9-10, pp. 924-941, 2003.

[13] W. Perruquetti and J. P. Barbot, Sliding Mode Control in Engineering, Marcel Dekker, New York, NY, USA, 2002.

[14] A. Damiano, G. L. Gatto, I. Marongiu, and A. Pisano, "Secondorder sliding-mode control of dc drives," IEEE Transactions on Industrial Electronics, vol. 51, no. 2, pp. 364-373, 2004.

[15] S. P. Bhat and D. S. Bernstein, "Finite-time stability of continuous autonomous systems," SIAM Journal on Control and Optimization, vol. 38, no. 3, pp. 751-766, 2000.

[16] S. P. Bhat and D. S. Bernstein, "Geometric homogeneity with applications to finite-time stability," Mathematics of Control, Signals, and Systems, vol. 17, no. 2, pp. 101-127, 2005.

[17] A. Levant, A. Pridor, R. Gitizadeh, I. Yaesh, and J. Z. Ben-Asher, "Aircraft pitch control via second-order sliding technique," Journal of Guidance, Control, and Dynamics, vol. 23, no. 4, pp. 586-594, 2000.

[18] Y. B. Shtessel, I. A. Shkolnikov, and A. Levant, "Smooth secondorder sliding modes: missile guidance application," Automatica, vol. 43, no. 8, pp. 1470-1476, 2007.

[19] C. Pukdeboon, "Finite-time second-order sliding mode controllers for spacecraft attitude tracking," Mathematical Problems in Engineering, vol. 2013, Article ID 930269, 12 pages, 2013.

[20] S. Yu, X. Yu, B. Shirinzadeh, and Z. Man, "Continuous finitetime control for robotic manipulators with terminal sliding mode," Automatica, vol. 41, no. 11, pp. 1957-1964, 2005.

[21] J. A. Moreno and M. Osorio, "Strict Lyapunov functions for the super-twisting algorithm," IEEE Transactions on Automatic Control, vol. 57, no. 4, pp. 1035-1040, 2012. 


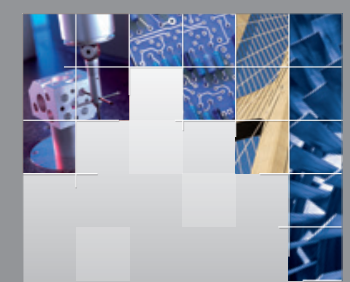

\section{Enfincering}
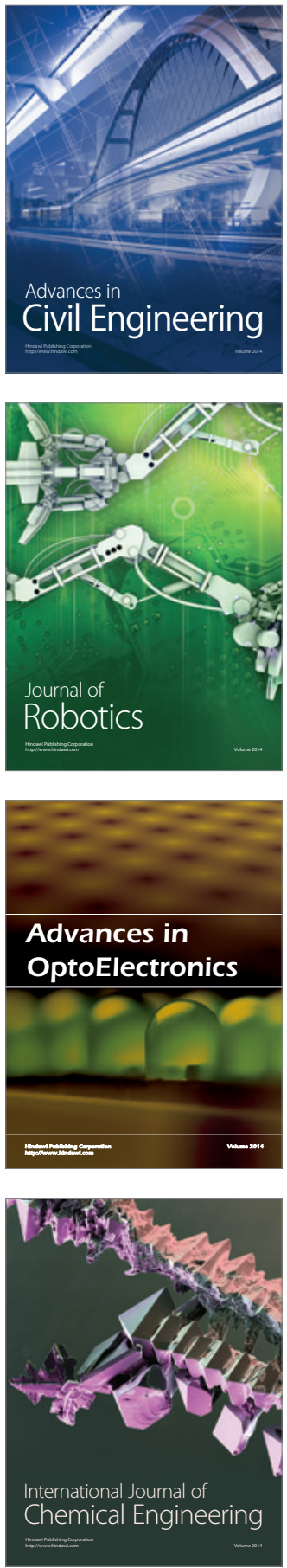

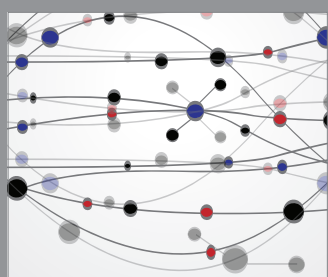

The Scientific World Journal

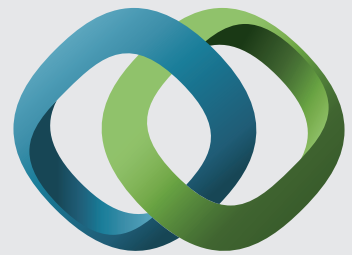

\section{Hindawi}

Submit your manuscripts at

http://www.hindawi.com
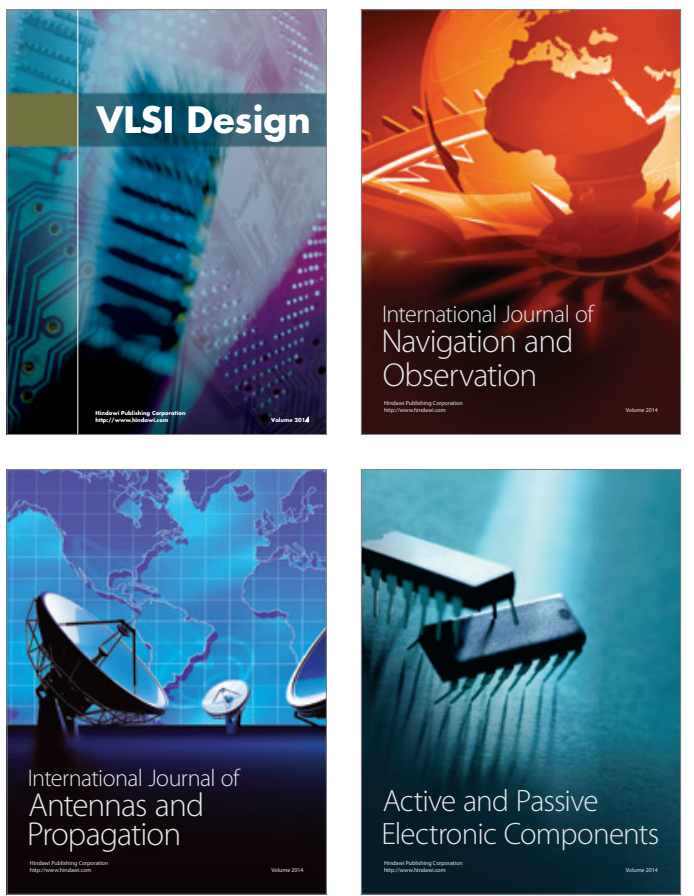
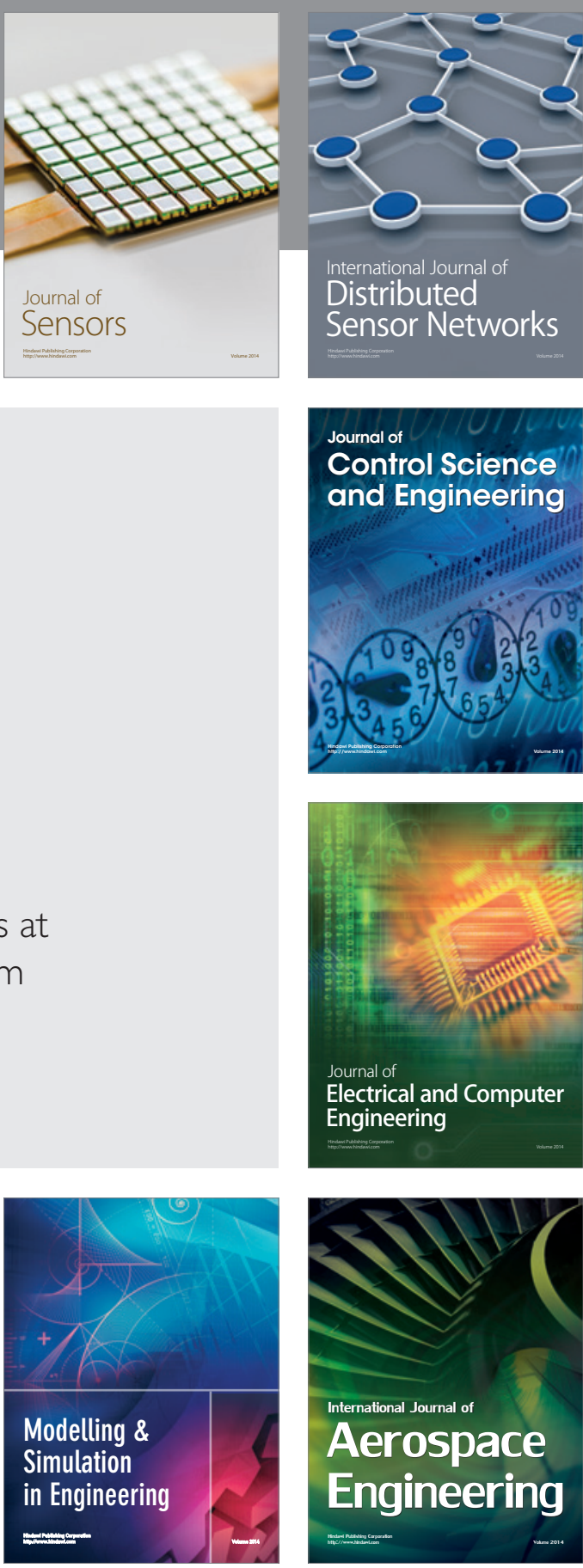

International Journal of

Distributed

Sensor Networks

Journal of

Control Science

and Engineering
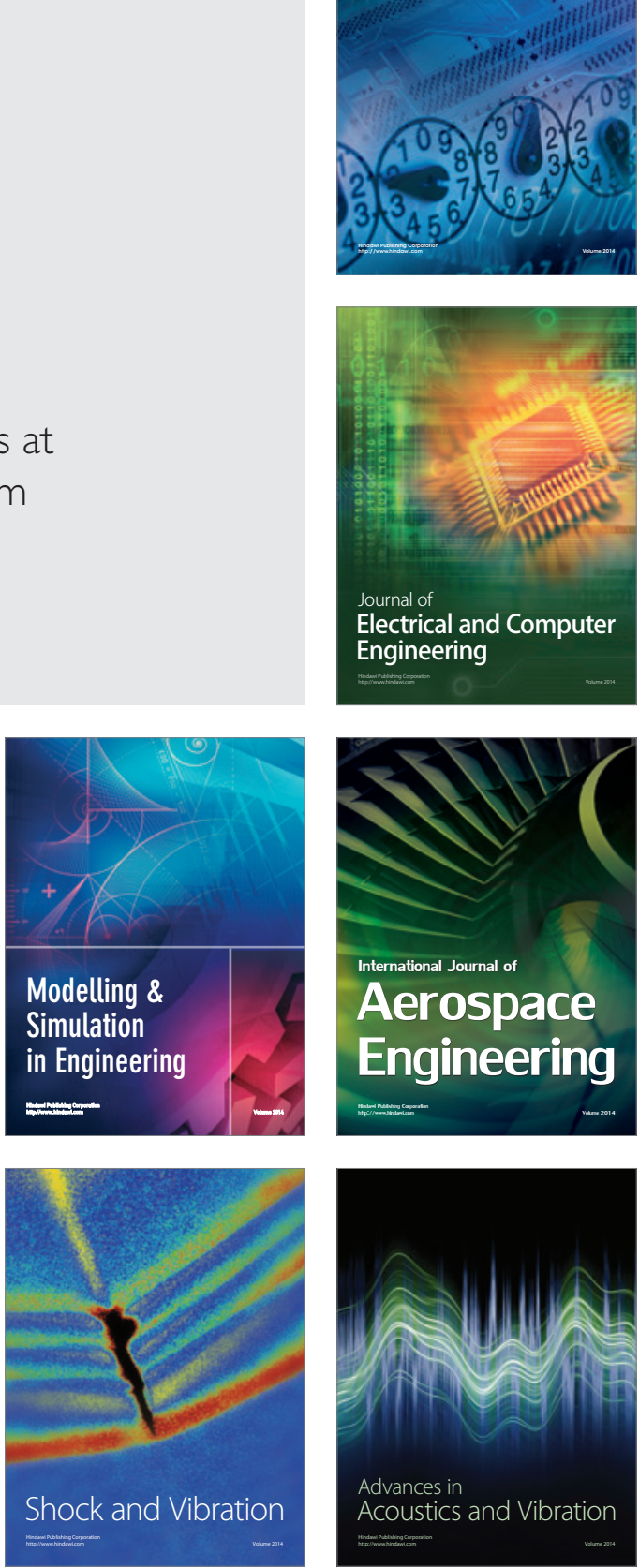